\title{
Road Grade Estimation for Look-ahead Vehicle Control
}

\author{
Per Sahlholm* Karl Henrik Johansson ** \\ * Scania CV AB, SE-151 87 Södertälje, Sweden (Tel: \\ +46-8-553-89129; e-mail: per.sahlholm@scania.com) \\ ** Royal Institute of Technology (KTH), SE-100 44, Stockholm, \\ Sweden
}

\begin{abstract}
Look-ahead cruise controllers and other advanced driver assistance systems for heavy duty vehicles require high precision digital maps. This contribution presents a road grade estimation algorithm for creation of such maps based on Kalman filter fusion of vehicle sensor data and GNSS positioning information. The algorithm uses data from multiple traversals of the same road to improve previously stored road grade estimates. Measurement data from three test vehicles and six road traversals has been used to evaluate the quality of the obtained road grade estimate compared to a known reference.
\end{abstract}

Keywords: Kalman filtering techniques in automotive control; Automotive system identification and modelling; General automobile/road-environment strategies

\section{INTRODUCTION}

Modern heavy duty vehicles (HDV) employ several electronic control systems which utilize vehicle and environment state information to increase efficiency, safety and comfort. The road grade is one state which heavily influences the longitudinal dynamics and energy flow in a heavy duty vehicle. It is used in engine and gearbox controllers to help meet the instantaneous power demand while keeping fuel consumption and environmental impact as low as possible.

The current state of the vehicle is commonly obtained through various on-board sensors. Advance knowledge, or look ahead, of future key influences on the vehicle enables new control algorithms to improve overall vehicle performance. As an example knowledge of the road grade about one kilometer ahead of the vehicle makes it possible to automatically adjust the speed ahead of up- and downhill sections and thus conserve fuel without increasing trip time. The preview road grade information can also be utilized when determining if a gearshift should be performed or the state of some energy buffer changed.

In order to reap the benefits described there has to be some way of knowing attributes related to the road ahead. This can be accomplished by using a global navigation satellite system (GNSS) receiver in combination with a digital map. GNSS receivers are already commonplace in vehicles, as are digital maps used for navigation. The road grade is currently not generally available in navigation maps, and has to be obtained by other means. One method is to use on-board sensors to estimate the road grade and create a map as the vehicle drives down the road. If a road is driven frequently, many estimates of the road grade can be obtained. These can be used to increase confidence in the created map. This contribution investigates properties of a proposed method for road grade estimation. The method combines road grade estimates based on standard mounted on-board sensors and a GPS receiver from many overlapping road traversals into a road grade map. Each time a known road is driven again the map can be updated. The method is tested with three types of HDVs, seen in Figure 1.
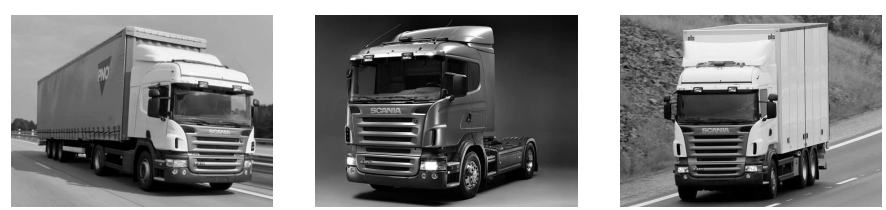

Fig. 1. The vehicle types used for verification. Starting from the left a tractor-semitrailer combination (A), tractor only (B), and rigid truck (C) were used.

\subsection{Related Work}

The potential for improved energy efficiency through speed optimization based on future road grade has recently been treated by e.g. Lattemann et al. [2004], Terwen et al. [2004], Hellström et al. [2007], Fröberg and Nielsen [2007]. Knowledge of future energy needs combined with new auxiliary units which enable increased power comsumption scheduling over time can improve total energy efficiency, as explored in Pettersson and Johansson [2006/05/In this context the future road grade is assumed to be known, for example from a map. Automatic map generation ideas have been described by Schroedl et al. [2004] and Brüntrup et al. [2005]. These contributions do not however specifically address road grade maps, or the possibility to use a vehicle model and driveline sensors to improve accuracy.

Many different methods for estimating the road grade can be found in the literature. One approach is to use a sensor directly related to the grade. This is used for 
example in Bae et al. [2001] where the grade is determined using a GPS receiver which give both a vertical and horizontal velocity. The road grade can then be found through the ratio of the velocities. Such a method relies heavily on the existence of a high quality GPS signal, something which is not always available. The idea of using vehicle sensor information to find the road grade has been explored in Lingman and Schmidtbauer [2001] where a Kalman filter is used to process a measured or estimated propulsion force or estimated retardation force and a measured velocity. A similar method, where the grade is estimated using Recursive Least Squares based on a simple motion model has been suggested by Vahidi et al. [2005]. These methods have the advantage of not needing any extra sensors, such as the GPS, but then neither provide the extra bias compensation or easy inclusion of data from multiple road traversals. Earlier treatments of the proposed grade estimation method can be found in Sahlholm et al. [2007b,a].

\subsection{Contribution}

This paper introduces a method for HDVs to estimate the road grade using only standard mounted sensors and a GPS receiver. Two implementations are presented, one based on a non-linear vehicle model and extended Kalman filtering and one based on a piecewise linear model and a standard Kalman filter. The method includes a systematic way of improving the current grade estimate using new passes over a know road segment. Incremental improvements are made possible by the use of spatial sampling and storage of the estimated error covariance matrix for the current road grade estimate. Grade estimates obtained under good conditions have a higher weight in the final estimate than those that are created under greater uncertainty. The storage requirement for a particular road will not grow as new measurements are incorporated. A step by step illustration of the effects of adding new measurements is presented. The grade estimation method detects and handles disturbances caused by GPS unavailability and driving events which change the vehicle dynamics. The proposed method is evaluated using three test vehicles driven a total of six times over the same test road segment. The obtained final grade estimate compares favorably to one acquired from specialized road grade measurement equipment.

\subsection{Outline}

The paper is organized as follows. Section 2 describes the the road grade estimation method by introducing the vehicle model, two different filtering approaches, smoothing and data fusion. It also explains the experimental setup. Results are given in section 3 , and the paper ends with conclusions and a discussion in section 4 .

\section{METHODOLOGY}

A non-linear vehicle model and an extended Kalman filter (EKF) are used to estimate the road grade. To investigate the effect of the nonlinearity, and obtain a linear model for further analysis, a piecewise constant linear vehicle model is also used for comparison. The road grade estimates from six test runs on highway E4 south of Södertälje, Sweden have been merged using a the proposed method.

\subsection{Vehicle Model and Measurements}

The first step of the road grade estimation method involves the integration of drive line sensors with GPS data. A longitudinal vehicle model is used to relate the various sensor signals to the road grade. A known vehicle mass and engine load at a particular gear together with the vehicle speed makes it possible to calculate the road grade. The most important forces which affects the vehicle are shown in Figure 2. The quantities are generally time varying, time

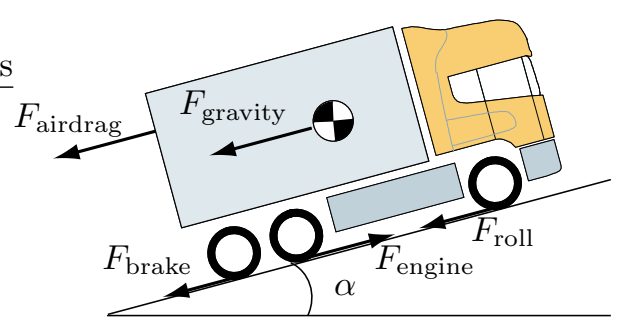

Fig. 2. Longitudinal forces acting on the vehicle.

has been left out of the equations for clarity. $F_{\text {engine }}=$ $\frac{i_{\mathrm{t}} i_{\mathrm{f}} \eta_{\mathrm{t}} \eta_{\mathrm{f}}}{r_{\mathrm{w}}} M$ is the net engine force. Knowledge of the selected gear yields the gear ratio $i_{\mathrm{t}}$ and the efficiency $\eta_{\mathrm{t}}$ from tables. The final gear ratio $i_{\mathrm{f}}$, efficiency $\eta_{\mathrm{f}}$ and wheel radius $r_{\mathrm{w}}$ are known vehicle constants. The engine torque measurement $M$ is obtained from the engine management system. $F_{\text {airdrag }}=\frac{1}{2} c_{\mathrm{w}} A_{\mathrm{a}} \rho_{\mathrm{a}} v^{2}$ is known through the measured vehicle speed $v$ together with the constants air drag coefficient $c_{\mathrm{w}}$, vehicle frontal area $A_{\mathrm{a}}$, and air density $\rho_{\text {air }}$. A very simple model $F_{\text {roll }}=m g c_{\mathrm{r}}$ gives the rolling resistance from the vehicle mass $m$, gravity $g$, and coefficient of rolling resistance $c_{\mathrm{r}}$. The road grade $\alpha$ enters the model through the gravity induced force $F_{\text {gravity }}=m g \sin \alpha$. The brake force $F_{\text {brake }}$ is excluded from the model since it is generally unknown in a standard HDV, its influence is considered at a later stage. The total dynamic vehicle mass is expressed as $m_{\mathrm{t}}=\frac{J_{\mathrm{w}}}{r_{\mathrm{w}}^{2}}+$ $m+\frac{i_{\mathrm{t}}^{2} i_{\mathrm{f}}^{2} \eta_{\mathrm{t}} \eta_{\mathrm{f}} J_{\mathrm{e}}}{r_{\mathrm{w}}^{2}}$ where $J_{\mathrm{w}}$ and $J_{\mathrm{e}}$ represent the inertia of the engine and the wheels respectively. Newton's laws of motion are used to attain a time relation between forces and velocity change.

A GPS receiver provides a three dimensional position (latitude, longitude, and altitude) together with a signal indicating the number of satellites used for the position fix. The vehicle speed and the road grade are used to calculate the time derivative of the altitude and thus provides a link between the GPS and the vehicle model. The changes in road grade are not modeled and the engine torque is regarded as an input signal $u(t)=M(t)$. Put together

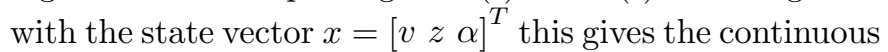
time vehicle and road model $\dot{x}(t)=f(x)$ with the dynamic equations given in (1). More details on the vehicle model formulation can be found in Kiencke and Nielsen [2003]. 


$$
\begin{aligned}
\dot{v}(t) & =\frac{1}{m_{\mathrm{t}}}\left(F_{\text {engine }}\right. \\
& \left.-F_{\text {airdrag }}-F_{\text {roll }}-F_{\text {gravity }}\right) \\
\dot{z}(t) & =v(t) \sin \alpha(t) \\
\dot{\alpha}(t) & =0
\end{aligned}
$$

In order to easily obtain estimates at specific spatial locations rather than time instants a spatially sampled version of the model is derived through the relation

$$
\frac{\partial v(t)}{\partial t}=\frac{\partial v(t)}{\partial s(t)} \underbrace{\frac{\partial s(t)}{\partial t}}_{v(t)} .
$$

The continuous model is then discretized with the distance step $\Delta s$ for use in the Kalman filter based state estimation. The discretized model is given in (2).

$$
\underbrace{\left[\begin{array}{c}
v_{k} \\
z_{k} \\
\alpha_{k}
\end{array}\right]}_{x_{k}}=\underbrace{\left[\begin{array}{c}
v_{k-1}+\Delta s \Delta v_{k} \\
z_{k-1}+\Delta s \sin \alpha_{k-1} \\
\alpha_{k-1}
\end{array}\right]}_{f_{k}\left(x_{k-1}, u_{k}\right)}+\underbrace{\left[\begin{array}{c}
w_{k}^{v} \\
w_{k}^{h} \\
w_{k}^{\alpha}
\end{array}\right]}_{w_{k}}
$$

The rate of change in velocity from the previous sample point is given by $(3)$.

$$
\begin{aligned}
\Delta v_{k}= & \underbrace{\frac{r_{\mathrm{w}} i_{\mathrm{t}} i_{\mathrm{f}} \eta_{\mathrm{t}} \eta_{\mathrm{f}}}{J_{\mathrm{w}}+m r_{\mathrm{w}}^{2}+i_{\mathrm{t}}^{2} i_{\mathrm{f}}^{2} \eta_{\mathrm{t}} \eta_{\mathrm{f}} J_{\mathrm{e}}}}_{c_{1}} \frac{M_{k-1}}{v_{k-1}} \\
& -\underbrace{\frac{\frac{1}{2} r_{\mathrm{w}}^{2} c_{\mathrm{w}} A_{\mathrm{a}} \rho_{\mathrm{a}}}{J_{\mathrm{w}}+m r_{\mathrm{w}}^{2}+i_{\mathrm{t}}^{2} i_{\mathrm{f}}^{2} \eta_{\mathrm{t}} \eta_{\mathrm{f}} J_{\mathrm{e}}}}_{c_{2}} v_{k-1} \\
& -\underbrace{\frac{r_{\mathrm{w}}^{2} m g}{J_{\mathrm{w}}+m r_{\mathrm{w}}^{2}+i_{\mathrm{t}}^{2} i_{\mathrm{f}}^{2} \eta_{\mathrm{t}} \eta_{\mathrm{f}} J_{\mathrm{e}}}}_{c_{3}} \frac{1}{v_{k-1}}\left(c_{\mathrm{r}}+\sin \alpha(s)\right)
\end{aligned}
$$

It can be noted that the values of $c_{1}, c_{2}$, and $c_{3}$ depend on the vehicle parameters as well as the selected gear. The presence of the efficiencies $\eta_{\mathrm{t}}$ and $\eta_{\mathrm{f}}$ also make the expression (3) dependent on whether the net engine torque is positive or negative.

To evaluate the influence of the nonlinearity in the vehicle model a piecewise constant version is derived. The linearization is done around an equilibrium, and reiterated at gear changes and depending on the direction of power flow in the drive line. When the engine is powering the vehicle the gearbox and final drive losses lead to lower total power at the wheels than at the engine. During coasting the engine acts as a break force and the situation is reversed, requiring adaptation of the model. Each gear and power flow direction will lead to a different mode, denoted by $m$, with a specific required torque to maintain a constant speed, and equilibrium in the model. The linear discretized model around the equilibrium $x_{m}$ is given by the system transition matrix $F_{m}$ and the input model $G$ according to

$$
\tilde{x}_{k+1}=F_{m} \tilde{x}_{k}+G \tilde{u}_{k}
$$

where $\tilde{x}=x-x_{m}$ is the state relative to the linearization point , $\tilde{u}=M-M_{m}$ is the relative engine torque. The transition matrix is given by $F_{m}=I+\left.\frac{\partial f}{\partial x}\right|_{x=x_{m}} \Delta s$. Using the model from before $F_{m}$ and $G$ became

$$
F_{m}=\left[\begin{array}{ccc}
1+\Delta v_{m} \Delta s & 0 & -\frac{c_{3}}{v_{m}} \cos \alpha_{m} \Delta s \\
0 & 1 & \cos \alpha_{m} \Delta s \\
0 & 0 & 1
\end{array}\right], G=\left[\begin{array}{c}
\frac{c_{1}}{v_{m}} \Delta s \\
0 \\
0
\end{array}\right]
$$

where $\Delta v_{m}=-c_{1, m} * \frac{M_{m}}{v_{m}^{2}}-c_{2, m}+\frac{c_{3}}{v_{m}^{2}}\left(c_{r}+\sin \alpha_{m}\right)$. The constants $c_{1, m}, c_{2, m}$, and $c_{3, m}$ are obtained by setting the gear ratio $i_{t}$ and efficiencies $\eta_{t}$ and $\eta_{f}$ to the values appropriate for each mode. The equilibrium point $x_{m}$ is obtained by choosing $v_{m}=80 \mathrm{~km} / \mathrm{h}, z_{m}=0 \mathrm{~m}, \alpha_{m}=0$. This gives $M_{m}=\frac{c_{2} v_{m}^{2}+c_{3} c_{r}+c_{3} \sin \alpha_{m}}{c_{1}} \mathrm{~N}$.

Two states and the input torque $M$ are measured for the state estimation. The measured states are the vehicle velocity $v$ and the altitude $z$. This leads to a linear measurement equation (5)which can be used for both the linear and non-linear vehicle model representations.

$$
y_{k}=\underbrace{\left[\begin{array}{lll}
1 & 0 & 0 \\
0 & 1 & 0
\end{array}\right]}_{H_{k}}\left[\begin{array}{l}
v_{k} \\
z_{k} \\
\alpha_{k}
\end{array}\right]+\underbrace{\left[\begin{array}{c}
e_{k}^{\mathrm{v}} \\
e_{k}^{\mathrm{z}}
\end{array}\right]}_{e_{k}}
$$

\subsection{State Estimation}

Two different Kalman filters are used to estimate the road grade and other model states. The non-linear model is used together with an EKF, and the piecewise linear model with a standard Kalman filter (KF). The process and measurement noises in the vehicle model are updated depending on the characteristics of the driving situation and GPS position reliability.

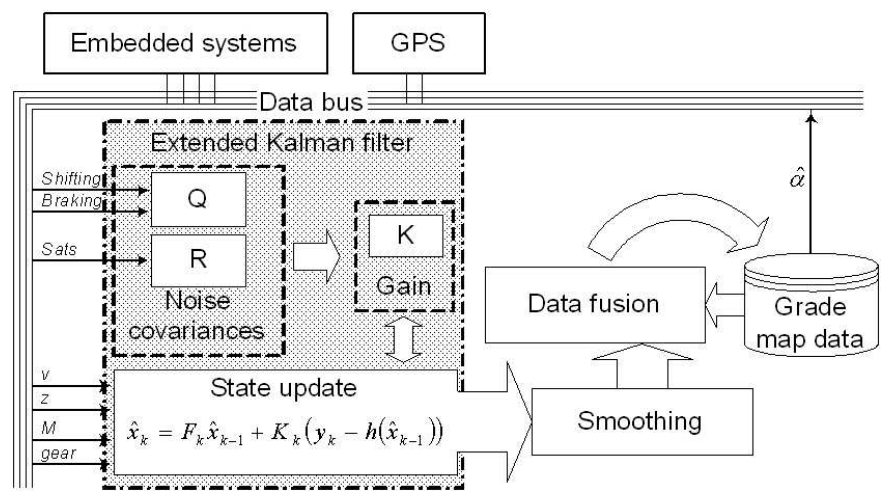

Fig. 3. Overview of the data filtering, smoothing and fusion of the proposed road grade estimation method.

Using the notation of the previous section the estimation model for the nonlinear EKF with a linear measurement equation is given by (6).

$$
\begin{aligned}
& x_{k}=f\left(x_{k-1}, u_{k}\right)+w_{k} \\
& y_{k}=H x_{k}+e_{k}
\end{aligned}
$$

Details on the Kalman filters can be found in Kailath et al. [2000]. In the EKF the nonlinear model is linearized around the current state at every time step. The obtained transition matrix $F_{k}$ is then used to complete the steps of the standard Kalman filter recursions. These recursions are described by two update steps: a time update and a measurement update. In the time update the system model is used to predict the future state of the system. Using the notation $\hat{x}_{k \mid k-1}$ to denote the quantity $\hat{x}$ at time $k$ based 
on information available up to time $k-1$ the time update is done according to $(7)$.

$$
\begin{aligned}
& \hat{x}_{k \mid k-1}=f\left(x_{k-1}, u_{k}\right) \\
& P_{k \mid k-1}=F_{k} P_{k-1 \mid k-1} F_{k}^{T}+Q_{k}
\end{aligned}
$$

Similarly to the piecewise linear model the transition matrix $F_{k}$ is defined to be the Jacobian $F_{k}=\left.\frac{\partial f}{\partial x}\right|_{\hat{x}_{k-1 \mid k-1}, u_{k}}$. $P_{k \mid k-1}$ is the estimated error covariance, and $Q_{k}=E\left[w_{k}^{2}\right]$ is the process noise covariance. After the time update the measurement at time $k$ is used in a measurement update to improve the estimate. The measurement update is described by (8).

$$
\begin{aligned}
K_{k} & =P_{k \mid k-1} H^{T}\left(H P_{k \mid k-1} H^{T}+R_{k}\right)^{-1} \\
\hat{x}_{k \mid k} & =\hat{x}_{k \mid k-1}+K_{k}\left(y_{k}-H \hat{x}_{k \mid k-1}\right) \\
P_{k \mid k} & =\left(I-K_{k} H\right) P_{k \mid k-1}
\end{aligned}
$$

Here $K_{k}$ is the Kalman gain, and $R_{k}=E\left[e_{k}^{2}\right]$ is the measurement noise covariance.

The piecewise constant linear model is filtered using a regular Kalman filter. At each mode change between different linearizations the final state of the old filter is used to initialize the new one. The linear system model in each mode is given by (9) where $\tilde{y}_{k}=y_{k}-H x_{m}$.

$$
\begin{aligned}
\tilde{x}_{k} & =F_{m} \tilde{x}_{k-1}+G \tilde{u}_{k}+w_{k} \\
\tilde{y}_{k} & =H \tilde{x}_{k}+e_{k}
\end{aligned}
$$

Which leads to the KF time update equations (10).

$$
\begin{aligned}
& \hat{x}_{k \mid k-1}=F_{m} \hat{x}_{k-1 \mid k-1}+G u_{k} \\
& P_{k \mid k-1}=F_{k} P_{k-1 \mid k-1} F_{k}^{T}+Q_{k}
\end{aligned}
$$

The measurement update equations are identical to the EKF case.

For this method the true process and noise covariances $R_{k}$ and $Q_{k}$ are not known from the start. Instead they are used as time varying design parameters to tune the filter to different driving situations. To simplify the design the noise covariance matrices were chosen to be diagonal. The diagonal elements are directly associated to the three model states and two measured quantities. For normal driving at a fixed gear $Q_{k}$ was tuned to give a filter with a time constant similar to the one used to produce our reference road grade estimate. $R_{k}$ was adjusted depending on the number of GPS satellites available. While other factors also affect the GPS position accuracy the number of satellites was the only relevant signal available from the satellite receiver used. When satellite coverage was lost a very high variance for was set for the altitude measurement, causing the grade estimate only to depend on vehicle signals. Driving events such as gearshifts and braking affect the vehicle in ways that are not covered by the relatively simple vehicle model given in (1). To account for this the process variance for the velocity state was increased during those events.

By running the data fusion step off line when complete road sections had been estimated it is possible to use smoothing to compensate for the filtering delay and include later measurements in the estimate for each data point. The Rauch-Tung-Striebel fixed point smoothing algorithm, introduced in Rauch et al. [1965], was used in this work. The smoothing was applied as a backwards recursion on the completed estimate of a road section. The final states, where $k=N$, of the filtered quantities were used to initialize the recursion. $P_{k}^{s}$ denotes the smoothed error covariance, $\hat{x}_{k}^{s}$ is the smoothed state estimate, and $K_{k}^{s}$ is the smoothing gain. The smoothing recursion is given by (11).

$$
\begin{aligned}
K_{k}^{s} & =P_{k \mid k} F_{k}^{T} P_{k+1 \mid k}^{-1} \\
\hat{x}_{k \mid N}^{s} & =\hat{x}_{k \mid k}+K_{k}^{s}\left(\hat{x}_{k+1 \mid N}^{s}-\hat{x}_{k+1 \mid k}\right) \\
P_{k \mid N}^{s} & =P_{k \mid k}+K_{k}^{s}\left(P_{k+1 \mid N}^{s}-P_{k+1 \mid k}\right) K_{k}^{s T}
\end{aligned}
$$

\subsection{Data Fusion}

In order to merge data from many passes over the same road segment a distributed data fusion method is used. The distributed approach has the important advantage that the data which has to be stored does not increase as additional measurements of known road segments are incorporated into the map. For each road segment, the map consists of the road related states (altitude $z$ and slope $\alpha$ ) and the associated estimated error covariance estimates for those states. Based on the map estimated error covariances and the estimated error covariances of a new smoothed estimate, an updated map is created each time a new measurement of a road segment becomes available. The new map becomes a weighted average of the two sources. Details on the data fusion algorithm (12) can be found in Gustafsson [2000].

$$
\begin{aligned}
P_{k}^{f} & =\left(\left(P_{k}^{1}\right)^{-1}+\left(P_{k}^{2}\right)^{-1}\right)^{-1} \\
\hat{x}_{k}^{f} & =P_{k}^{f}\left(\left(P_{k}^{1}\right)^{-1} \hat{x}_{k}^{1}+\left(P_{k}^{2}\right)^{-1} \hat{x}_{k}^{2}\right)
\end{aligned}
$$

$P_{k}^{f}$ is the resulting error covariance, $\hat{x}_{k}^{f}$ is the new slope estimate for the map. The quantities $P_{k}^{1}, P_{k}^{2}, \hat{x}_{k}^{1}$, and $\hat{x}_{k}^{2}$ are the source estimates and estimated error covariances. Initially both the source sets are smoothed results from individual measurement runs, after that one source will be the map (based on all previous runs), and one will be the new measurement to be incorporated.

\subsection{Experiment setup}

The proposed road grade estimation algorithm has been tested on highway E4 south of Södertälje in Sweden. Three test vehicles, representing the different types shown in Figure 1 were used. Important properties for the test vehicles are listed in Table 1. A total of six round-trip measurements were conducted. The different vehicles were driven on different days under varying weather conditions. Separate model parameters were used for each of the vehicles. The use of more than one vehicle type introduced a beneficial spread of the parameter errors. The measurements thus included some of the variations that would affect a real world system used in many separate vehicles.

Most of the signals needed for the road grade estimation are available on the CAN bus of stock production trucks. These are the vehicle speed, engine torque, current gear, gearshift status, and brake utilization. The CAN bus signals were recorded using a laptop. There was no GPS data available on the vehicle bus, instead an external VBOX GPS receiver with a CAN interface was used. The GPS data was logged using the same computer as 
Table 1. Key properties of the test vehicles used to collect experiment data. The total vehicle weight is given in tons.

\begin{tabular}{|c|c|c|c|c|}
\hline Vehicle & Configuration & Weight & Axles & Meas. \\
\hline A & Tractor and semi-trailer & $39 \mathrm{t}$ & 5 & $1,2,3$ \\
\hline B & Tractor & $13 \mathrm{t}$ & 2 & 4,5 \\
\hline C & Rigid truck & $21 \mathrm{t}$ & 3 & 6 \\
\hline
\end{tabular}

the vehicle data, which provided for easy and accurate synchronization with the vehicle data.

The described method is intended for highway use, where the wheel slip is relatively small and constant. The front wheel rotation sensor was used to determine the vehicle speed $v$, with a compensation factor calculated from GPS velocity measurements during good signal conditions. The engine torque was reported from the engine management system, based on fuel injection data. The quality of this signal is usually reasonable, even though variations between individual engines, and over the life of a single engine are present. The gearbox management system relayed the current gear, and if a shift was in process. The truck brake system reported when either the auxiliary or wheel brakes were in use, but could only give a torque estimate for the auxiliary brake.

The absolute position obtained from the GPS was used to synchronize data from the different measurements in order to complete the data fusion. First a reference point was chosen in one of the measurements. The closest points in the other measurements were then used as their respective starting points. From the starting point the traveled distance information in each measurement was used to resample all signals to a common distance vector. With common distance indexing it was then possible to complete the road grade estimation and data fusion steps.

\section{RESULTS}

Road grade estimates obtained from regular highway driving at the normal cruising speed are very good. Without the GPS altitude measurements the vehicle model and measured signals give an estimated grade which has a bias due to modeling errors. The bias is reduced when the GPS altitude measurement is introduced as an independent correction in the filter. Using more than one road traversal and more than one vehicle improves the final grade estimate. All result figures presented share the same distance scale for easy cross-referencing. A reference grade profile obtained from a specialized measurement vehicle is used to evaluate the estimates. Figure 4 shows the agreement of the final grade estimate with the reference for a part of the test road. The numerical standard deviation for the smoothed estimates from the individual traversals is also shown. The part of the test road shown in Figure 4 contains a downhill section, from $1000 \mathrm{~m}$ to $2600 \mathrm{~m}$. Around $2000 \mathrm{~m}$ vehicle A needs to apply the brakes in order to avoid over speeding. During braking the torque affecting the vehicle is unknown. The process noise term in $Q_{k}$ corresponding to the velocity state is increased in order to decrease the reliance on the model and increase the estimated slope error covariance. The braking in the downhill section leads to a lower quality overall grade estimate, which shows up as an increased confidence mar-

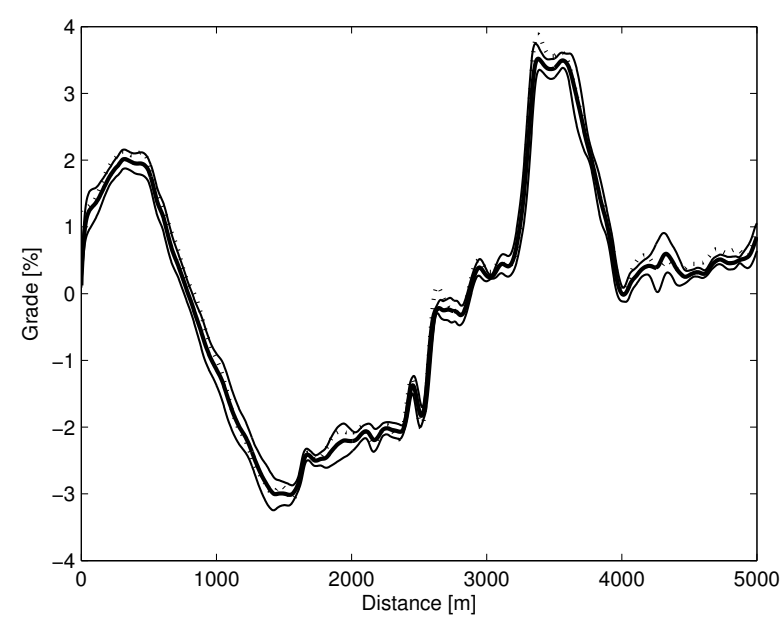

Fig. 4. The final grade estimate calculated through data fusion based on six road traversals (solid) agrees well with the reference grade profile (dashed) from a specialized measurement vehicle. The numerical one standard deviation confidence interval around the final grade estimate at each sample point is also shown (thin lines).

gin. Figure 5 shows the increased estimated slope error covariance for measurement two $P_{k(3,3)}^{s}$ as a result of the braking together with the effects of gearshifts mandated by the hill around $4000 \mathrm{~m}$.

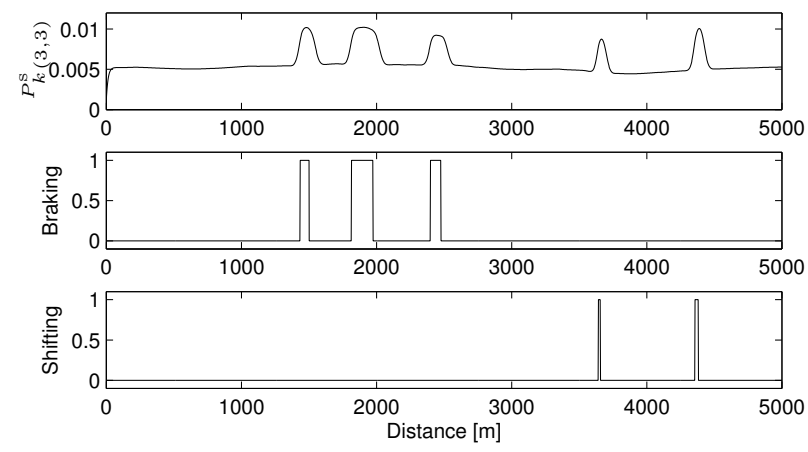

Fig. 5. The influence on the estimated slope error covariance $P_{k(3,3)}^{s}$ (top figure) from braking (logical signal in the middle figure) and shifting (logical signal in the bottom figure) during measurement two is shown. When the estimated error covariance is high for one grade estimate that data carries less weight in the data fusion step.

\subsection{Data Fusion}

Figure 6 shows a comparison of the smoothed estimates from all six traversals with the final grade estimate and the reference grade profile for the most challenging part of the test road, the downhill section from $1300 \mathrm{~m}$ to $2300 \mathrm{~m}$. The mean value at each sample point is also shown to illustrate the effect of the data fusion step. When using data from more than one vehicle, collected on different days, many of the attributes determining how well the vehicle model fits will vary. If the variation is centered 


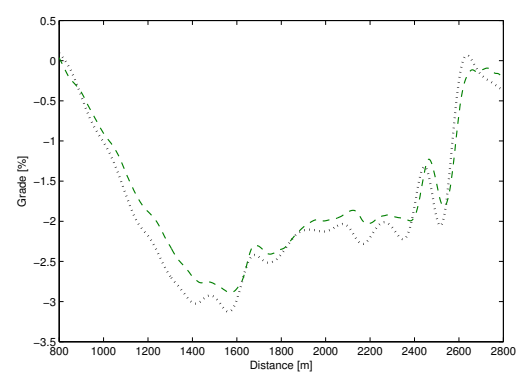

(a) The first measurement forms a road grade map by itself. Estimation errors cause it to differ from the reference road grade.

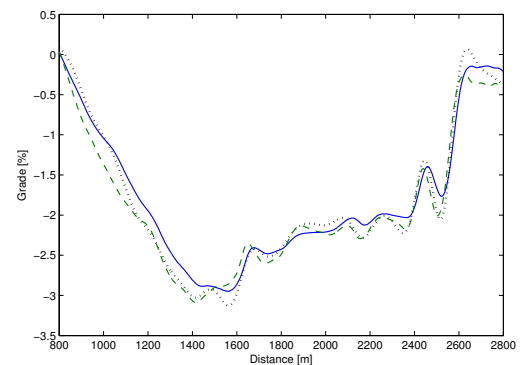

(d) The fourth estimate, obtained using vehicle $\mathrm{B}$, shows larger differences. This is probably in part due to different model parameter errors.

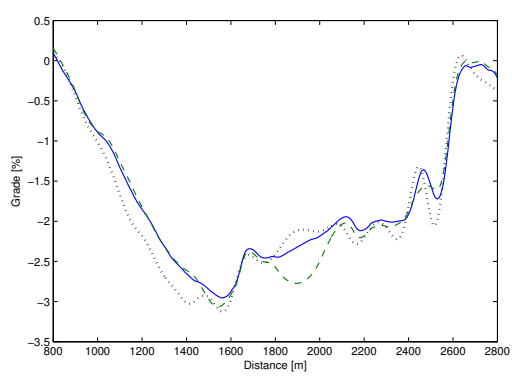

(b) When a second measurement is added to the one in (a) a new road grade map is obtained. The large disturbance in measurement two at $1900 \mathrm{~m}$ has a relatively low weight in the data fusion, due to high uncertainty.

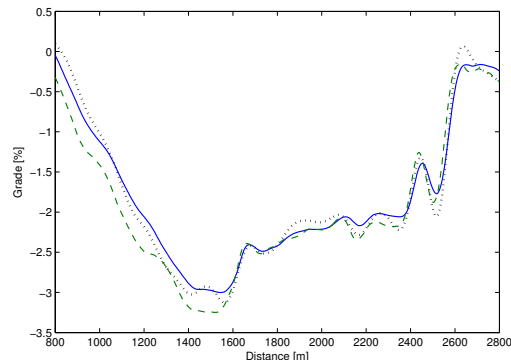

(e) Estimate five is based on vehicle B, just the one in $(d)$.

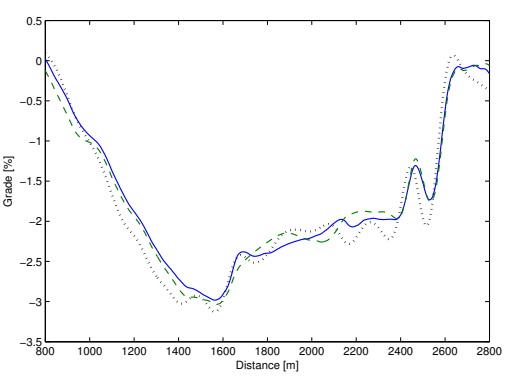

(c) The third estimate from vehicle A does not differ much from the map based on the previous two road traversals.

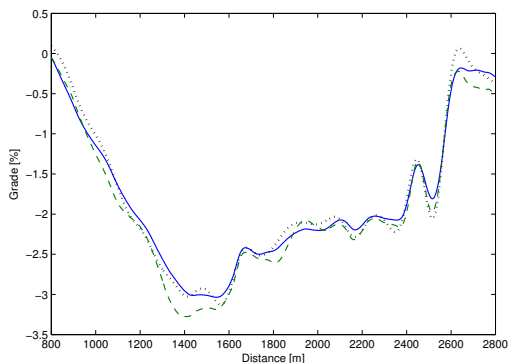

(f) When the sixth estimate, recorded with vehicle $\mathrm{C}$, has been added the map shows good agreement with the reference road grade.

Fig. 7. As more measurements are added the road grade map is improved. The sub-figures (a)-(f) show the progression as six measurements are combined into one road grade map. Each figure shows the latest measurement (dashed), the road grade map based on all measurements added so far (solid) and the reference road grade (dotted).

around the value assumed in the processing, using many road traversals can significantly improve the final result. The grade maps resulting from the progressive inclusion of the six recorded road traversals can be seen in Figure 7.

\subsection{Linearization Effects}

The results from using the piecewise constant linear model instead of the time-varying non-linear model indicated only marginal changes in the estimated slope for the investigated road segment. A comparison of road grade estimates obtained with the two methods is given in Figure 8 . The main non-linearity in the vehicle model, for the magnitude of slopes considered, is in the velocity. During most of the test road measurements the velocity of the measuring vehicle was near the linearization point $80 \mathrm{~km} / \mathrm{h}$. Other tests with larger velocity deviations, together with frequent linear model switching when changing to lower gears due to a rapid speed decrease suggested larger differences between the two methods. The number of mode switches between different linear models can be decreased by neglecting the efficiencies $\eta_{t}$ and $\eta_{f}$, this would make engine powered and coasting modes identical and cut the number of required modes by one half to the number of possible gears. It is however not possible to use a constant linear model, since gear changes heavily affects the matrices $F$ and $G$ through the change in the ratio $i_{g}$.

\section{CONCLUSIONS AND DISCUSSION}

For the investigated test cases the piecewise linear model performs in a similar manner to the time-varying nonlinear model for the task of estimating highway grades. This opens up possibilities both to lower the computational requirements to get more insight into how various events affect the filter. One such planned extension is the estimation of the true process and measurement noise covariances $Q$ and $R$. Better synchronization of the different measurement runs based on the absolute positions at more instants than the start of measurement has the potential to reduce slope errors caused by misalignment between measurements. This is particularly true when the grade changes quickly. Misalignment occurs primarily because of different systematic odometry errors in vehicles, and varying traveled paths on the road surface.

Measurements from more vehicles and more road passes will make it possible to deduce more precisely what grade estimation errors are random and reduced with additional data, and which are systematic and more crucial to deal with in the method. Already at this stage the proposed method is feasible for collecting road grade data of sufficient quality for model predictive control based energy optimization of the vehicle longitudinal motion. 


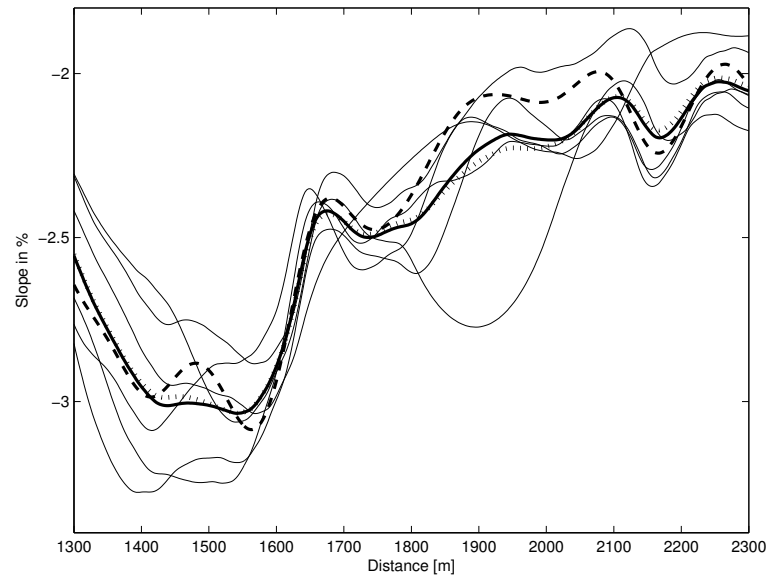

Fig. 6. The final merged road grade estimate (solid) is shown with the reference grade profile (dashed) and the mean value of all smoothed estimates (dotted). The smoothed estimates from the individual traversals are also included (thin lines). This is a magnification of the most challenging part of the test road. Measurement two is particularly at odds with the rest around the braking instances shown in Figure 5 (at $1500 \mathrm{~m}$ and $1900 \mathrm{~m})$. This is due to a combination of poor GPS coverage and the effect of the braking at those points. Due to the higher estimated error covariance these artifacts have less influence on the fused estimate than on the mean of all the estimates.

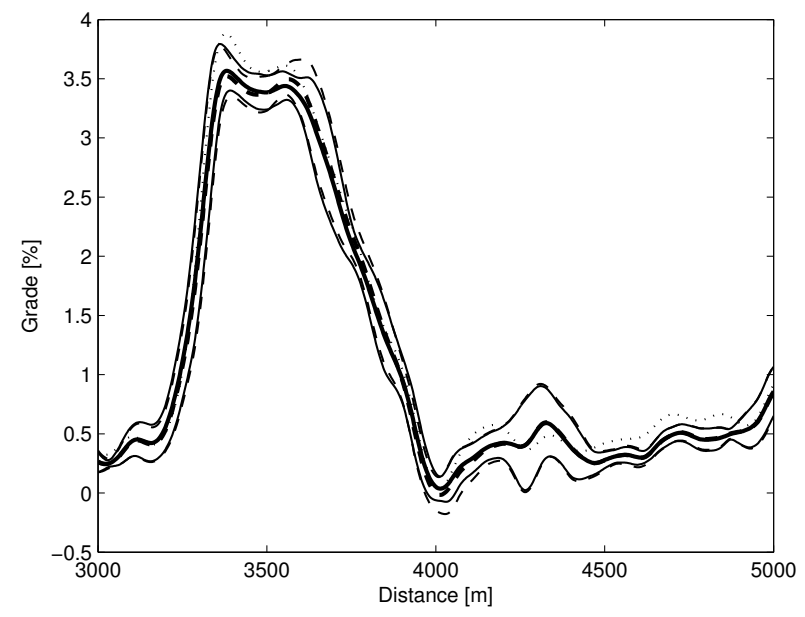

Fig. 8. The final grade estimate based on the non-linear model (solid) with confidence interval (thin lines) is shown together with the one based on the piecewise constant linear model (dashed). The reference grade is also shown (dotted thin line). The differences between the two methods are slight, and significantly smaller than the deviation from the reference grade.

\section{ACKNOWLEDGEMENTS}

This work is partially supported by Scania CV AB, the Swedish Program Board for Automotive Research (IVSS), and by the European Commission through the Network of Excellence HYCON.

\section{REFERENCES}

H. S. Bae, J. Ruy, and J. Gerdes. Road grade and vehicle parameter estimation for longitudinal control using GPS. In Proceedings of IEEE Conference on Intelligent Transportation Systems, San Francisco, CA, 2001.

R. Brüntrup, S. Edelkamp, S. Jabbar, and B. Scholz. Incremental map generation with GPS traces. In Proceedings of IEEE Intelligent Transportation Systems, Vienna, Austria, 2005.

A. Fröberg and L. Nielsen. Fuel optimal speed profiles. Fifth IFAC Symposium on Advances in Automotive Control, Monterey Coast CA, USA, 2007.

F. Gustafsson. Adaptive filtering and change detection. John Wiley \& Sons, LTD, Chichester, 2000.

E. Hellström, M. Ivarsson, J. Åslund, and L. Nielsen. Look-ahead control for heavy trucks to minimize trip time and fuel consumption. Fifth IFAC Symposium on Advances in Automotive Control, Monterey Coast CA, USA, 2007.

T. Kailath, A.H. Sayed, and B. Hassibi. Linear estimation. Upper Saddle River, NJ, 2000.

U. Kiencke and L. Nielsen. Automotive Control Systems. Springer Verlag, Berlin, 2003.

F. Lattemann, K. Neiss, S. Terwen, and T. Connolly. The predictive cruise control-a system to reduce fuel consumption of heavy duty trucks. SAE Technical Paper Series, 2004.

P. Lingman and B. Schmidtbauer. Road slope and vehicle mass estimation using Kalman filtering. In Proceedings of the 19th IAVSD Symposium, Copenhagen, Denmark, 2001.

N. Pettersson and K.H. Johansson. Modelling and control of auxiliary loads in heavy vehicles. International Journal of Control, 79(5):479 - 95, 2006/05/. ISSN 0020-7179.

H.E. Rauch, F. Tung, and C.T. Striebel. Maximum likelihood estimates of linear dynamic systems. AIAA Journal, 3(8):1445 - 1450, 1965.

P. Sahlholm, H. Jansson, and K.H. Johansson. Road grade estimation results using sensor and data fusion. 14th World Congress on Intelligent Transport Systems, Beijing, China, 2007a.

P. Sahlholm, H. Jansson, E. Kozica, and K.H. Johansson. A sensor and data fusion algorithm for road grade estimation. Fifth IFAC Symposium on Advances in Automotive Control, Monterey Coast CA, USA, 2007b.

S. Schroedl, K. Wagstaff, S. Rogers, P. Langley, and C. Wilson. Mining gps traces for map refinement. Data Mining and Knowledge Discovery, 9:59-87, 2004.

S. Terwen, M. Back, and V. Krebs. Predictive powertrain control for heavy duty trucks. In Proceedings of IFAC Symposium on Advances in Automotive Control, Salerno, Italy, 2004.

A. Vahidi, A. Stefanopolou, and H. Peng. Recursive least squares with forgetting for online estimation of vehicle mass and road grade: Theory and experiments. Journal of Vehicle System Dynamics, 43:31-57, 2005. 